\title{
miR-125b-5p inhibits cell proliferation, migration, and invasion in hepatocellular carcinoma via targeting TXNRD1
}

Shengni Hua ${ }^{1}$, Yingyao Quan ${ }^{1}$, Meixiao Zhan ${ }^{1}$, Huaxin Liao ${ }^{2^{*}}$, Yong Li ${ }^{1^{*}}$ and Ligong Lu ${ }^{1^{*}}$ (D)

\begin{abstract}
Background: Thioredoxin reductase 1 (TXNRD1) is an antioxidant enzyme reportedly overexpressed in hepatocellular carcinoma (HCC); however, the detailed function and mechanisms of TXNRD1 in HCC remain obscure. In this study, we investigated the miR-125b-5p-specific regulation of TXNRD1 levels and its effect on HCC cells.

Methods: We detected miR-125b-5p levels in human HCC tissue samples through quantitative reverse transcription polymerase chain reaction (qRT-PCR), and in vitro experiments were employed to investigate the effect of miR-125b-5p on HCC cell proliferation, migration, and invasion. Additionally, we examined miR-125b-5p-mediated changes in TXNRD1 levels by qRT-PCR and western blotting, and a dual luciferase-reporter assay was conducted to confirm direct targeting of the $3^{\prime}$ untranslated region of TXNRD1 mRNA by miR-125b-5p.
\end{abstract}

Results: miR-125b-5p expression was reduced in HCC tissues relative to that in matched para-carcinoma tissues; this finding was verified in HCC cohorts from the Gene Expression Omnibus and The Cancer Genome Atlas. Additionally, low miR-125b-5p expression was associated with poor prognosis in HCC patients, and gene-set enrichment analysis indicated that miR-125b-5p levels were associated with HCC proliferation and metastasis. As predicted, overexpressing miR-125b-5p restrained the proliferation, migration, and invasion of Huh7 and SK-Hep-1 cells and forced expression of the miR-125b-5p-downregulated TXNRD1 mRNA and protein levels in HCC cells. Moreover, dual luciferasereporter assays revealed that miR-125b-5p targets TXNRD1 to directly regulate its expression, whereas TXNRD1 overexpression abolishes the inhibitory effect of miR-125b-5p on HCC cell proliferation, migration, and invasion.

Conclusions: These results demonstrated miR-125b-5p as a tumor suppressor in HCC through its inhibition of TXNRD1, thereby suggesting it as a potential target for the clinical treatment of HCC.

Keywords: miR-125b-5p, Hepatocellular carcinoma, Proliferation, TXNRD1

\section{Background}

Liver cancer is reportedly the 6th most common cancer and the 4th most lethal cause of cancer-related death worldwide, with hepatocellular carcinoma (HCC) accounting for $\sim 80 \%$ of these cases [1]. Due to various pathogeneses involved in HCC tumor progression, the

\footnotetext{
*Correspondence: hliao@duke.edu; lorry5160@163.com; luligong1969@126.com

${ }^{1}$ Zhuhai Interventional Medical Center, Zhuhai Precision Medical Center, Zhuhai People's Hospital, Zhuhai Hospital Affiliated with Jinan University, Zhuhai 519000, China

${ }^{2}$ Biomedicine Institute, College of Life Science, Jinan University, Guangzhou 510632, China
}

heterogeneity of $\mathrm{HCC}$ cells, and the late stage during which HCC is usually identified, the prognosis of HCC patients is poor and, despite the recent discovery of subclinical molecular targets, the overall survival rate of HCC remains low [2]. Therefore, it is necessary to continue investigating the molecular mechanisms associated with HCC progression in order to identify additional prognostic indicators.

MicroRNAs (miRNAs) are small, noncoding RNAs that participate in the posttranscriptional regulation of gene expression [3], and accumulating data indicate that miRNAs play an important role in modulating multiple biological behaviors associated with cancer [4]. Moreover,

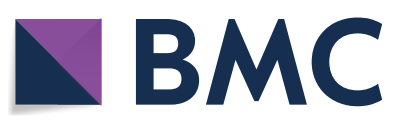

(c) The Author(s) 2019. This article is distributed under the terms of the Creative Commons Attribution 4.0 International License (http://creativecommons.org/licenses/by/4.0/), which permits unrestricted use, distribution, and reproduction in any medium, provided you give appropriate credit to the original author(s) and the source, provide a link to the Creative Commons license, and indicate if changes were made. The Creative Commons Public Domain Dedication waiver (http://creativecommons.org/ publicdomain/zero/1.0/) applies to the data made available in this article, unless otherwise stated. 
miRNA levels are correlated with neoplasm staging, tumor relapse, drug tolerance, and prognosis [5-7]. However, the mechanisms underlying the miRNA-specific regulation of tumor progression have not been completely investigated.

Aerobic organisms exhibit sophisticated antioxidation mechanisms in order to maintain a reducing environment in cells. Thioredoxin-1 (TRX1) is a key contributor to intracellular redox homeostasis and promotes energy and carbohydrate metabolism [8]. Mechanistically, TRX1 responds to reactive oxygen species produced by cellular respiration and metabolism and facilitates the reduction of other proteins at the cost of its own oxidation [9]. Thioredoxin reductase-1 (TXNRD1) is uniquely capable of utilizing electrons from NADPH to recover the reduced state of TRX1. Interestingly, TXNRD1 is upregulated in many human malignancies and promotes cancer progression [10], and attenuation of TXNRD1 levels effectively suppresses the growth of tumor cells [11].

In this study, we investigated the regulatory relationship between miR-125b-5p and TXNRD1 in HCC cells. Furthermore, we examined the effect of miR-125b-5p on the biological function of $\mathrm{HCC}$ cell lines.

\section{Methods \\ Clinical samples}

A total of 13 pairs of fresh $\mathrm{HCC}$ and matched adjacent tissues were acquired from patients undergoing their first hepatectomy and diagnosed with HCC between May 2016 and June 2018 at the Zhuhai People's Hospital, Jinan University (Zhuhai, China). None of the patients had received radiotherapy or chemotherapy prior to the hepatectomy. Ethics approval for this study was obtained from the Zhuhai People's Hospital Institutional Review Board with approval number [2016 trial (the institute) in subclause (10)].

\section{Cell culture}

Two human HCC cell lines (Huh7 and SK-Hep-1) were obtained from the Cell Bank of Type Culture Collection (Chinese Academy of Sciences, Shanghai, China). Cells were cultured with Dulbecco's modified Eagle's medium (Invitrogen, Carlsbad, CA, USA) containing 10\% fetal bovine serum (FBS; Invitrogen) and incubated at $37{ }^{\circ} \mathrm{C}$ with $5 \% \mathrm{CO}_{2}$.

\section{Gene- and miRNA-expression datasets}

Data regarding miR-125b-5p levels from $372 \mathrm{HCC}$ patients (follow-up information was included for 328 patients), and data regarding TXNRD1 levels from 351 HCC patients (concurrent data concerning miR-125b-5p levels were included for 347 patients) were obtained from The Cancer Genome Atlas (TCGA; https://tcga-data. nci.nih.gov/tcga/). Additionally, five microarray datasets (GSE22058, GSE54751, GSE21362, GSE12717, and GSE10694) were obtained from the Gene Expression Omnibus (GEO; http://www.ncbi.nlm.nih.gov/geo).

\section{Gene set enrichment analysis (GSEA)}

GSEA was performed to identify gene sets or pathways associated with miR-125b-5p levels in the TCGA dataset. The 347 samples, for which miRNA- and mRNAexpression profiles were available, were used for GSEA after being divided into two groups: miR-125b-5p high or low expression. The gene pool, including the RAMASWAMY metastasis-associated gene set (http://software. broadinstitute.org/gsea/msigdb/cards/RAMASWAMY_ METASTASIS_UP), was a gift from Zhongshan University (Guangzhou, China). We used GSEA software (v.2.0; http://www.broadinstitute.org/gsea), with $P<0.05$ considered statistically significant.

\section{miRNA transfection}

miR-125b-5p mimic/inhibitor was purchased from RiboBio (Guangzhou, China). A miR mimic/inhibitor was transfected into HCC cells upon their reaching 20\% to $30 \%$ confluence and using Lipofectamine RNAiMAX (Invitrogen) at a working concentration of $100 \mathrm{nM}$ according to manufacturer instructions. Cells were used for analysis after a $24-$ to 72 -h culture.

\section{Plasmid transfection}

Huh7, SK-Hep-1, and HEK293T cells were placed in a plate (Nest Biotechnology, Wuxi, China) and transfected with plasmids upon reaching $80 \%$ to $90 \%$ confluence using Lipofectamine 3000 (Invitrogen) according to manufacturer instructions, after with cells were used for analyses after $48 \mathrm{~h}$. The TXNRD1-expression plasmid and those harboring either wild-type (WT) or mutant versions of the TXNRD1 $3^{\prime}$ untranslated region (UTR) were obtained from ViGene Biosciences (Shandong, China).

\section{Luciferase-reporter assay}

The fragment of the TXNRD1 $3^{\prime}$ UTR containing the miR-125b-5p binding site (named WT) and fragment containing the site modified by site-directed mutagenesis (named Mutant) were cloned into the psiCHECK-2.0 vector (Promega, Madison, WI, USA). For the $3^{\prime}$ UTR dual-luciferase reporter assay, miR-125b-5p-overexpressing or -silenced HEK293 cells were co-transfected with WT or Mutant plasmids. Luciferase activity was detected using the luciferase reporter assay kit (Promega) at 72-h post-transfection. 


\section{Quantitative reverse transcription polymerase chain reaction (qRT-PCR)}

RNA was acquired using an RNA extraction kit (Invitrogen) according to manufacturer instructions, and qRT-PCR was performed using a SYBR Green PCR kit (Takara, Dalian, China), with glyceraldehyde 3-phosphate dehydrogenase and U6 used as internal controls for protein-coding genes and miR-125b-5p, individually. Primer specificity was verified prior to use, and assays were performed in triplicate. Primer sequences of primer are listed in Additional file 1: Table S1.

\section{Western blotting}

HCC cells were lysed on ice using radioimmunoprecipitation assay buffer (Beyotime, Nanjing, China) containing phosphatase and protease inhibitors (Roche, Basel, Switzerland). Total protein was quantitated using the Bradford assay (Thermo Fisher Scientific, Waltham, MA, USA). Equivalent protein lysates were subjected to $10 \%$ sodium dodecyl sulfate polyacrylamide gel electrophoresis and then transferred onto a polyvinylidene fluoride membrane that was blocked using 5\% nonfat milk for $40 \mathrm{~min}$ at $37^{\circ} \mathrm{C}$, followed by incubation at $4{ }^{\circ} \mathrm{C}$ for $>8 \mathrm{~h}$ with antibodies against TXNRD1 (Proteintech, Rosemont, IL, USA) and horseradish peroxidase-conjugated secondary antibodies (Cell Signaling Technology, Danvers, MA, USA). Peroxidase activity was visualized using an enhanced chemiluminescence substrate (BioRad Laboratories, Hercules, CA, USA).

\section{Cell Counting Kit (CCK)-8 assay}

Cells were placed in 96-well plates $(\sim 800$ cells/well; Nest Biotechnology, Jiangsu, China), and $10 \mu \mathrm{L}$ of CCK-8 solution (Dojindo Laboratories, Osaka, Japan) was added to each well at 24-, 48-, 72-, 96-, or 120-h post-treatment. After 2-h incubation in the dark, absorbance was detected at $450 \mathrm{~nm}$.

\section{5-ethynyl-2'-deoxyuridine (EdU)-incorporation assay}

Cells in 96-well plates at a density of 5000 cells/well were analyzed utilizing a Cell-Light EdU Apollo 567 kit (RiboBio) according to the manufacturer's instructions. We used an inverted fluorescence microscope (Olympus, Tokyo, Japan) to count cells in five random fields, with experiments performed in triplicate using independent samples.

\section{Transwell-migration and Boyden-invasion assays}

For the Transwell-migration assay, $\sim 1 \times 10^{5}$ cells suspended in $100 \mu \mathrm{L}$ of serum-free medium were placed in the upper layer of the Transwell (Corning Life Sciences, Lowell, MA, USA) while the lower layer received
$500 \mu \mathrm{L}$ of medium containing $10 \%$ FBS. We washed the inserts three times using phosphate-buffered saline, and fixed cells that adhered to the Transwell were incubated with methanol for $\sim 10 \mathrm{~min}$ at room temperature, followed by dying with crystal violet for $20 \mathrm{~min}$. Experiments were performed in triplicate. Boyden-invasion assay were performed using the same protocol as that for the cell-migration assay, but the membrane used was coated with $24 \mathrm{mg} / \mathrm{mL}$ Matrigel (BD Biosciences, Franklin Lakes, NJ, USA).

\section{Statistical analysis}

SPSS software (v.16.0; SPSS Inc., Chicago, IL, USA) was used for data analysis. Data represent the mean \pm standard error of the mean of at least three replicates. Student's $t$ test was used to evaluate differences between two groups, and one-way analysis of variance (ANOVA), followed by Dunnett's multiple comparison test was used to assess differences between multiple groups. The results of the CCK- 8 assay were evaluated using multi-way classification ANOVA, and overall and relapse-free survival rates were analyzed using the Kaplan-Meier method [12]. Correlations were analyzed with Pearson's correlation. A $P<0.05$ was considered statistically significant.

\section{Results}

miR-125b-5p levels are downregulated in HCC tissues and cell lines

We found that miR-125b-5p levels were significantly reduced in HCC tissues relative to those in precancerous non-tumor or normal liver tissues, according to data from five GEO datasets and a TCGA cohort (Fig. 1a-f). Additionally, qRT-PCR analysis showed that miR-125b-5p levels were lower in HCC tissues relative to those in corresponding non-cancerous tissues $(n=13 ; P=0.006)$ (Fig. 1g). Moreover, we confirmed that miR-125b-5p levels were lower in six HCC cell lines (SK-Hep-1, SMMC-7721, Huh7, HCCLM3, MHCC97H, and Hep3B) as compared with that in a normal liver cell line (HL-7702) (Fig. 1h). Furthermore, Kaplan-Meier survival analysis indicated that HCC patients exhibiting low miR-125b-5p expression had a poor prognosis in a TCGA cohort $(P=0.027)$ (Fig. 1i) and the GSE10694 dataset $(P=0.033)$ (Fig. 1j). GSEA showed miR-125b-5p expression was positively associated with gene signatures related to HCC-patient survival in the TCGA cohort (Fig. 1k). These results indicated that miR-125b-5p expression was downregulated in HCC samples and associated with HCC-patient prognosis. 

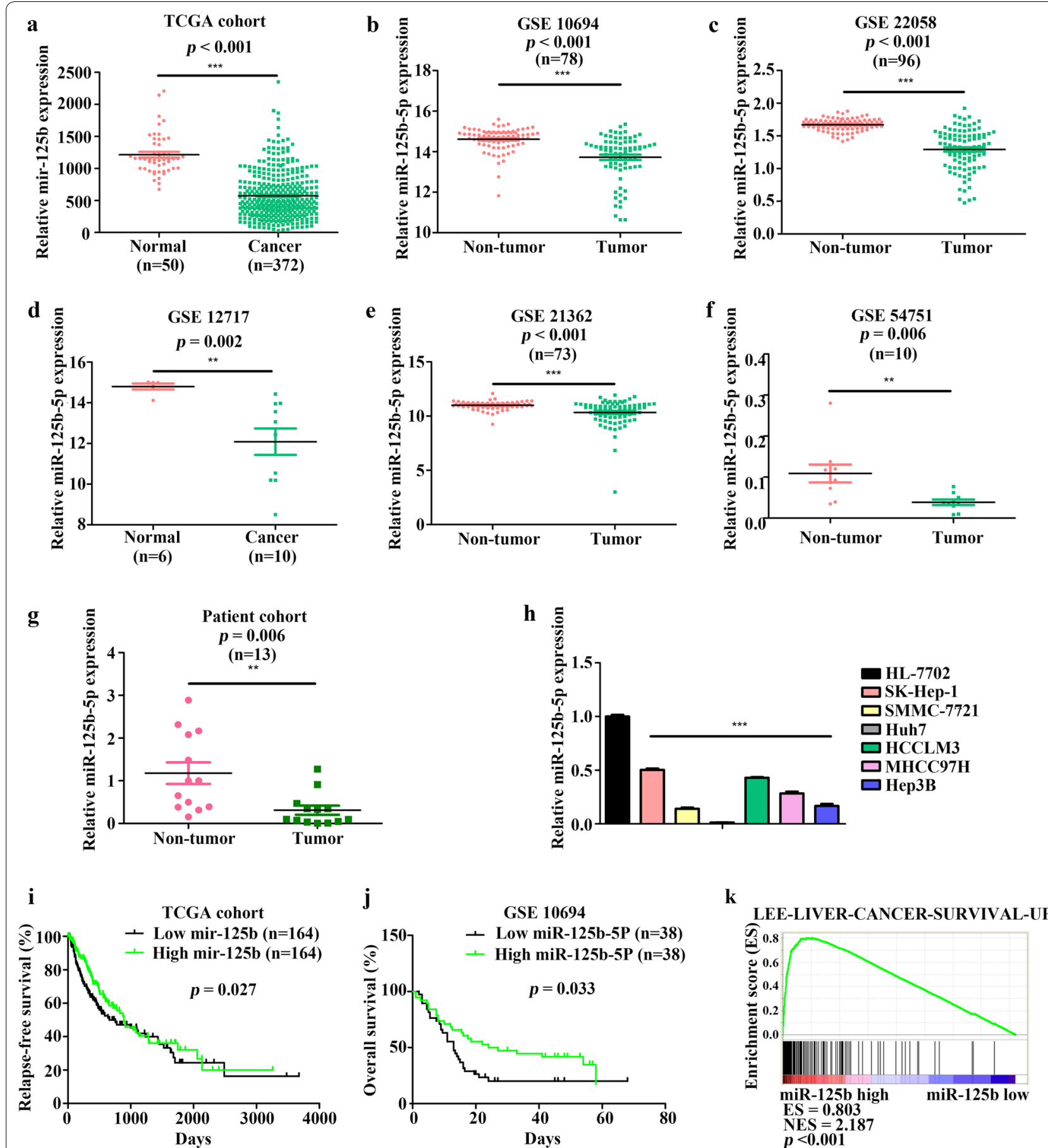

Fig. 1 miR-125b-5p levels are attenuated in HCC tissues. miR-125b-5p levels in HCC and non-tumor tissues in the a The Cancer Genome Atlas (TCGA) cohort and five Gene Expression Omnibus (GEO) datasets [b GEO series (GSE)10694, c GSE22058, d GSE12717, e GSE21362, and f GSE54751] $(P<0.001, P<0.001, P<0.001, P=0.002, P<0.001$, and $P=0.006$, respectively; Student's $t$ test). miR-125b-5p levels according to qRT-PCR in $\mathbf{g}$ an HCC cohort ( $n=13 ; P=0.006$; Student's $t$ test) and $\mathbf{h}$ normal liver cells and HCC cell lines. miR-125b-5p expression is associated with $\mathbf{i}$ relapse-free survival of HCC patients in the TCGA cohort and $\mathbf{j}$ the overall survival of HCC patients in the GSE10694 dataset $(P=0.027$ and $P=0.033$, respectively; Kaplan-Meier analysis). $\mathbf{k}$ HCC patients exhibiting elevated miR-125b-5p expression showed better overall survival than patients with low miR-125b-5p expression according to gene set enrichment analysis (GSEA) of the TCGA cohort (Enrichment score $=0.803, P<0.001$ ) 
miR-125b-5p inhibits HCC cell proliferation, migration, and invasion

To examine the biological roles of miR-125b-5p in HCC, we performed GSEA of the TCGA cohort, which indicated that miR-125b-5p might be involved in regulating HCC cell proliferation (Fig. 2a). We then transfected Huh7 and SK-Hep-1 cells with miR-125b-5p mimic/ inhibitor, followed by qRT-PCR assays to verify transfection efficiency (Fig. 2b, c). We found that miR-125b-5p overexpression suppressed Huh7 and SK-Hep-1 cell proliferation according to CCK-8 assays (Fig. 2d, e), whereas miR-125b-5p silencing had the opposite effect (Fig. 2f, g). Additionally, EdU-incorporation assays suggested that exogenous introduction or knockdown of miR-125b-5p in HCC cells reduced or increased the percentage of S-phase cells, respectively (Fig. 2h-k). Moreover, GSEA of the TCGA cohort revealed miR-125b-5p levels as negatively correlated with metastasis-related gene sets associated with HCC (Fig. 3a). Furthermore, Transwell and Boyden assays showed that miR-125b-5p upregulation attenuated the migratory ability and invasiveness of Huh7 and SK-Hep-1 cells (Fig. 3b-d), whereas miR-125b-5p knockdown resulted in the opposite effects (Fig. 3e-g), suggesting that miR-125b-5p inhibited the migration and invasion of HCC cells. These data indicated that ectopic expression of miR-125b-5p inhibited the proliferation, migration, and invasion of HCC cells.

\section{TXNRD1 is a direct target of miR-125b-5p}

To determine the mechanisms associated with the inhibitory effects of miR-125b-5p on HCC cell proliferation, migration, and invasion, we used the online website TargetScan (http://www.targetscan.org/) and miRDB (http:// www.mirdb.org/) to predict potential miR-125b-5p targets. Following literature review, we investigated targets reportedly upregulated in HCC according to the TGCA cohort, including TXNRD1, protein phosphatase $\mathrm{Mg}^{2+}$ / $\mathrm{Mn}^{2+}$-dependent-1F, and $\mathrm{Cbl}$ proto-oncogene $\mathrm{B}$. qRTPCR analysis of their mRNA levels in miR-125b-5poverexpressing or silenced HCC cells revealed that only TXNRD1 was regulated by miR-125b-5p (Fig. 4a, b). Moreover, western blot analysis of the same cells showed decreased or increased TXNRD1 levels in SK-Hep-1 and Huh7 cells depending on miR-125b-5p overexpression or knockdown, respectively (Fig. 4c, d). These results suggested that TXNRD1 might be targeted by miR-125b-5p.

To verify this hypothesis, we co-transfected luciferasereporter plasmids harboring either a WT or mutant version of the TXNRD1 $3^{\prime}$ UTR with the miR-125b-5p mimic/inhibitor into HEK293T cells. We observed reduced luciferase activity in $3^{\prime}$ UTR WT-but not mutant-transfected cells overexpressing miR-125b-5p, whereas cells harboring silenced miR-125b-5p displayed the opposite effects, indicating that miR-125b-5p directly modulated TXNRD1 levels by targeting its $3^{\prime}$ UTR (Fig. 4e, f). Furthermore, analysis of HCC cohorts for TCGA showed that TXNRD1 level were upregulated in HCC samples and negatively correlated with miR125b-5p expression (Fig. 4g, h). Moreover, HCC patients exhibiting elevated TXNRD1 levels showed a poor prognosis in the TCGA cohort $(P=0.022)$ (Fig. 4i). These data suggested TXNRD1 as a direct target of miR-125b-5p.

\section{miR-125b-5p modulates $\mathrm{HCC}$ cell proliferation, migration, and invasion by targeting TXNRD1}

Because TXNRD1 is directly targeted by miR-125b-5p, we presumed that TXNRD1 also mediates miR-125b-5p function. To test this hypothesis, we overexpressed TXNRD1 in HCC cells transfected with the miR-125b-5p mimic (Fig. 5a, b), and found that TXNRD1 overexpression reversed miR-125b-5p-mediated suppression of HCC cell proliferation, migration, and invasion according to EdU-incorporation, Transwell, and Boyden assays, respectively (Fig. $5 \mathrm{c}-\mathrm{h}$ ). These results indicated that miR125b-5p modulated the proliferation, migration, and invasion of HCC cells by targeting TXNRD1 (Fig. 6).

\section{Discussion}

In this study, we found that miR-125b-5p expression was attenuated in HCC tissues according to the analysis of five GEO datasets and a TCGA cohort, suggesting its possible involvement in regulating HCC progression. Although one study indicated its role in tumor progression [13], the overwhelming majority of reports suggest that miR-125b-5p as a tumor suppressor inhibits multiple biological functions in a variety of tumors, including those associated with HCC [14-19]. Additionally, previous studies report that miR-125b-5p suppresses HCC proliferation and metastasis by regulating the expression of downstream signaling molecules [17-22]. The findings of present study confirmed, for the first time, that miR-125b-5p targets TXNRD1 to inhibit HCC cell proliferation, migration, and invasion by downregulating TXNRD1 expression.

To identify potential miR-125b-5p targets in HCC cells, we screened predicted targets forecasted as upregulated in HCC tissues according to a TCGA cohort and reported as cancer-promoting genes in HCC in the literature. qRT-PCR analysis and co-transfection of HCC cells with a miR-125b-5p mimic or inhibitor 


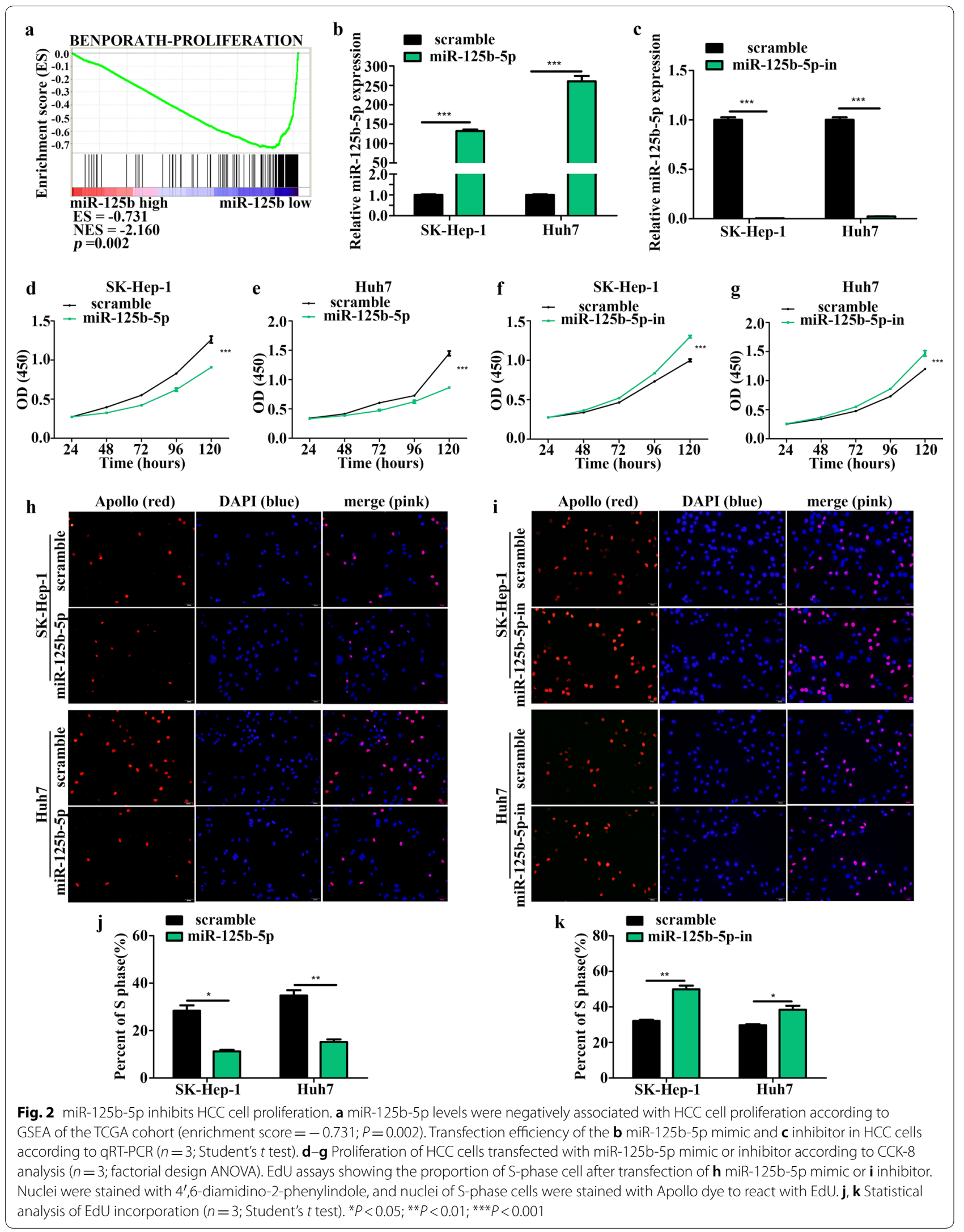



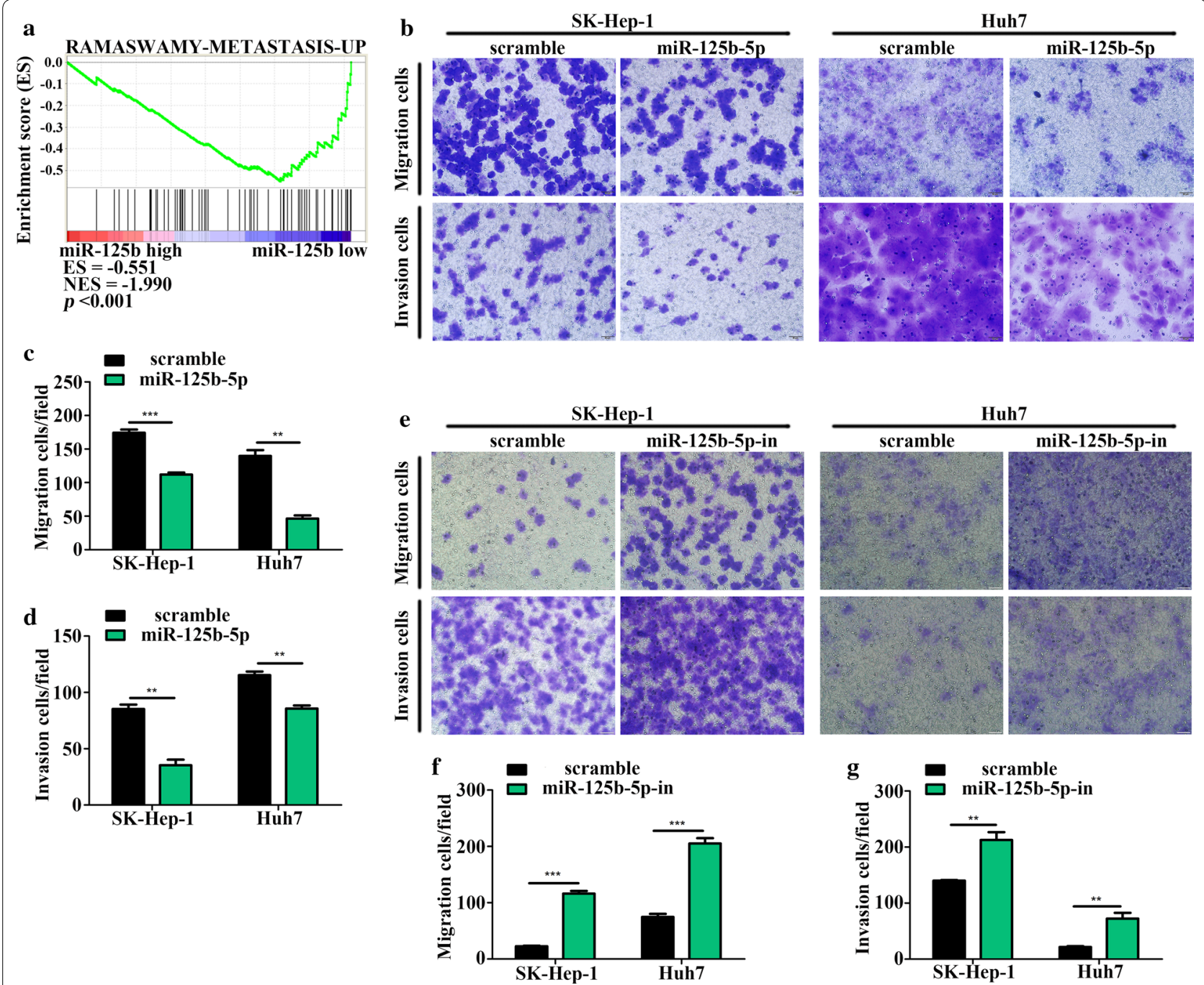

Fig. 3 miR-125b-5p inhibits HCC cell migration and invasion. a miR-125b-5p expression was negatively associated with metastasis according to GSEA of the TCGA cohort (enrichment score $=-0.551 ; P<0.001$ ). $\mathbf{b}$ HCC cell migration and invasion were evaluated in cells transfected with the miR-125b-5p mimic and using Transwell and Boyden assays. c, d Statistical analysis of Transwell and Boyden assay results $(n=3$; Student's $t$ test). e HCC cell migration and invasion were evaluated in cells transfected with miR-125b-5p inhibitor and using Transwell and Boyden assays. $\mathbf{f}, \mathbf{g}$ Statistical analysis of Transwell and Boyden assay results $\left(n=3\right.$; Student's $t$ test). ${ }^{*} P<0.05$; ${ }^{*} P<0.01 ;{ }^{* *} P<0.001$

revealed TXNRD1 as the target. Previous studies identified TXNRD1 as a regulator of endocellular oxidative stress and a promoter of tumor development [23, 24], with irreversible suppression of cytoplasmic TXNRD1 levels demonstrated as a novel direction for cancer treatment [11, 25, 26]. Additionally, studies reported that TXNRD1 levels are upregulated in HCC and negatively correlated with HCC-patient prognosis [27, 28]. In the present study, our data demonstrated that TXNRD1 promoted the proliferation, migration, and invasion of HCC cells, which provided insight into the previously unknown molecular mechanisms associated with TXNRD1 in HCC. Metabolic analysis showed that TXNRD1 is crucial for the final step in the synthesis 


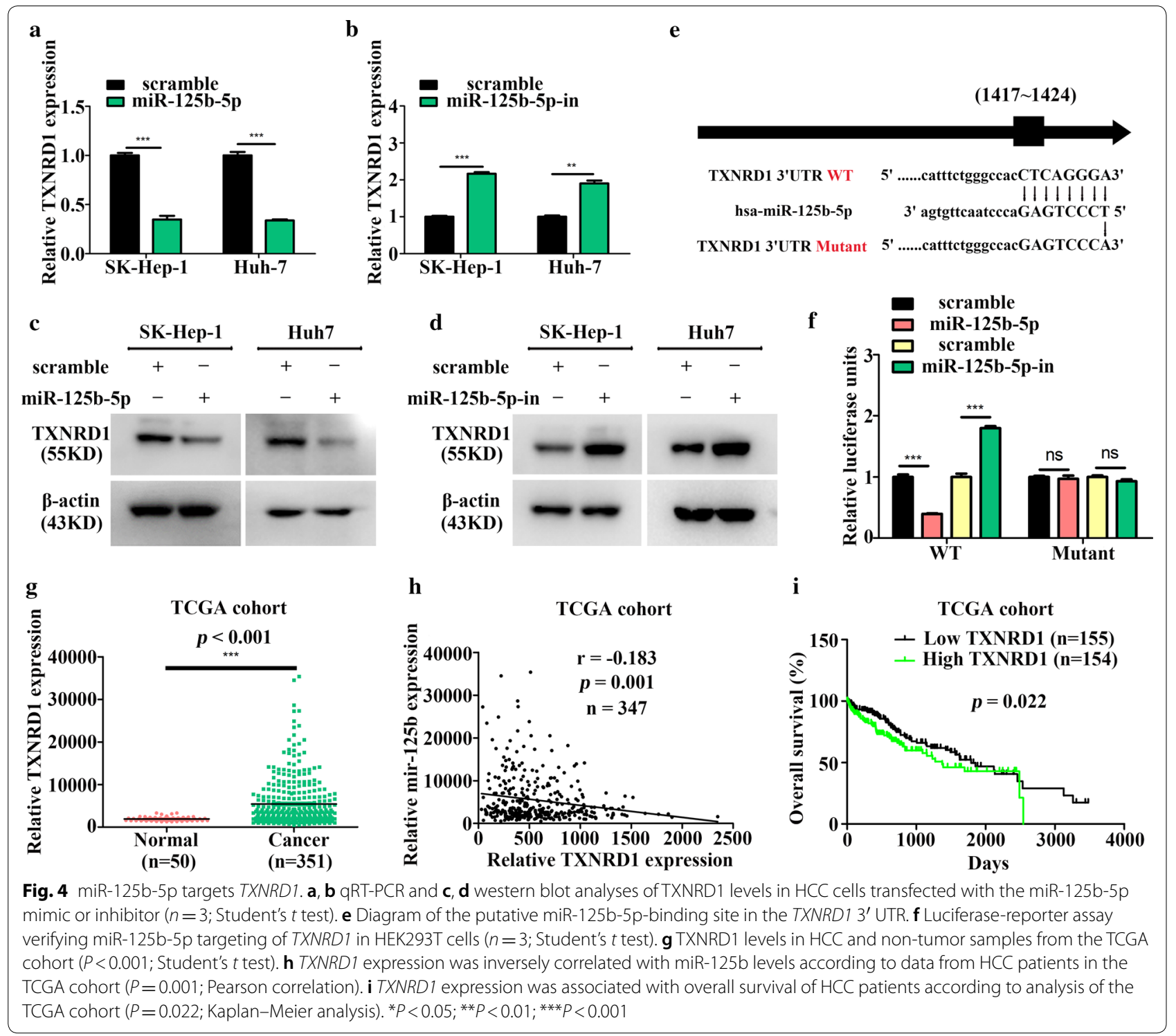

of nucleic acids by providing reductive equivalents to ribonucleotide reductase [29], and failure to maintain sufficient stores of $2^{\prime}$-deoxyribonucleotides resulted in damaged DNA and cell cycle arrest in TXNRD1-deficient $\mathrm{T}$ cells [30]. These findings and our results from the present study suggest that the mechanisms underlying TXNRD1 regulation of HCC status might be associated with oxidative stress or nucleotide biosynthesis; however, further studies are required to clarify these roles.

Here, we demonstrated the tumor-suppressive property of miR-125b-5p via its targeting of TXNRD1, which broadens our understanding of the molecular mechanism associated with miR-125b-5p-related functions in HCC cells. Nevertheless, the mechanism underlying 

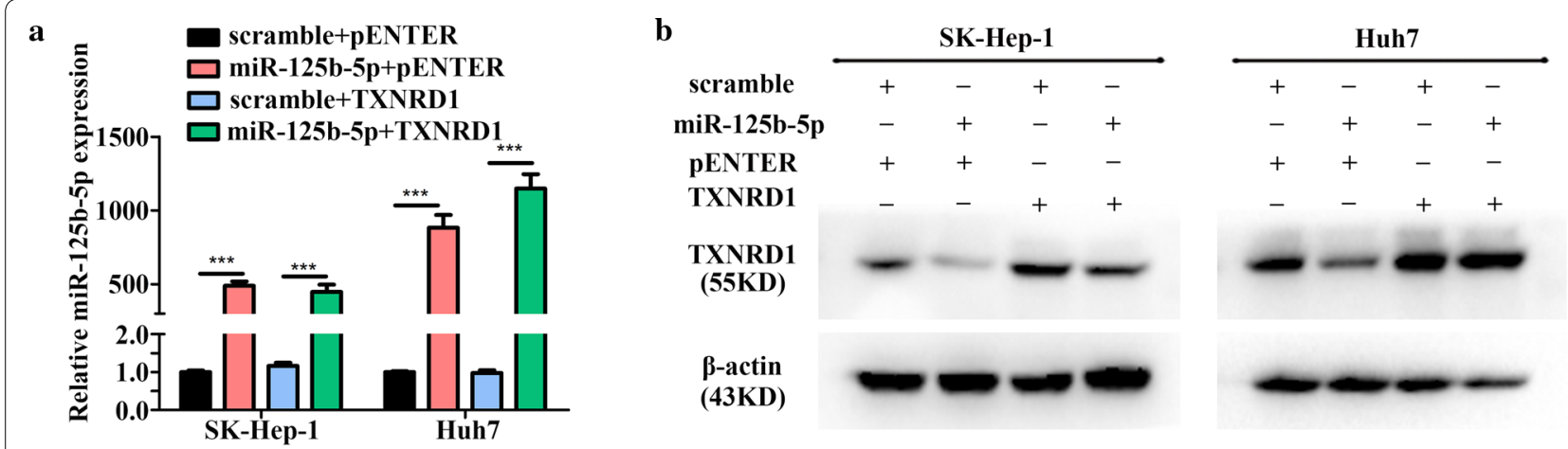

c scramble+pENTER miR-125b-5p+pENTER scramble+TXNRD1 miR-125b-5p+TXNRD1
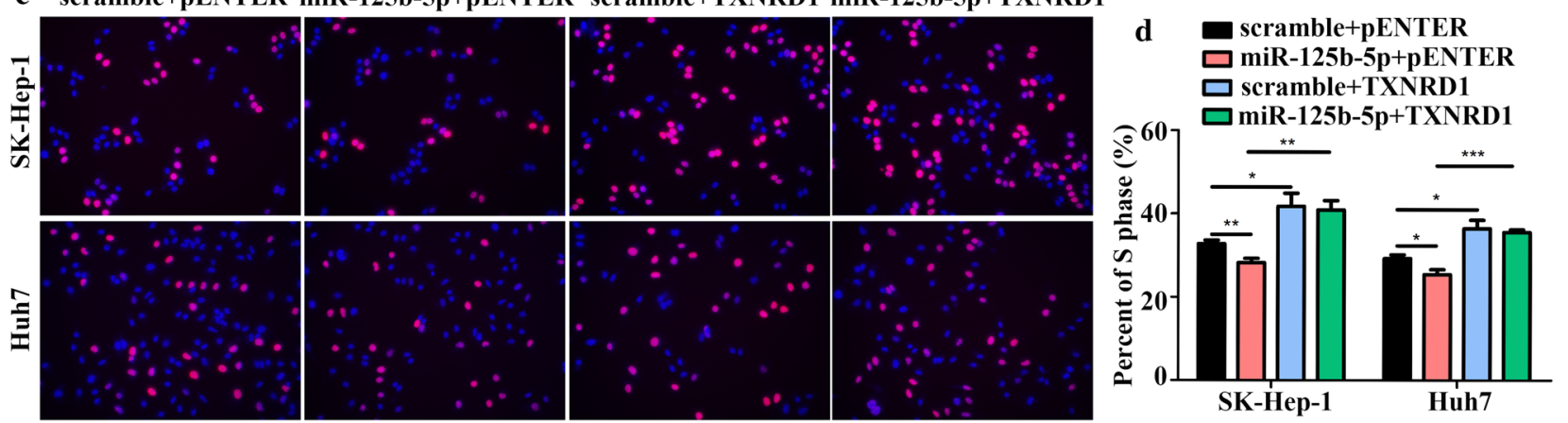

e scramble+pENTER miR-125b-5p+pENTER scramble+TXNRD1 miR-125b-5p+TXNRD1
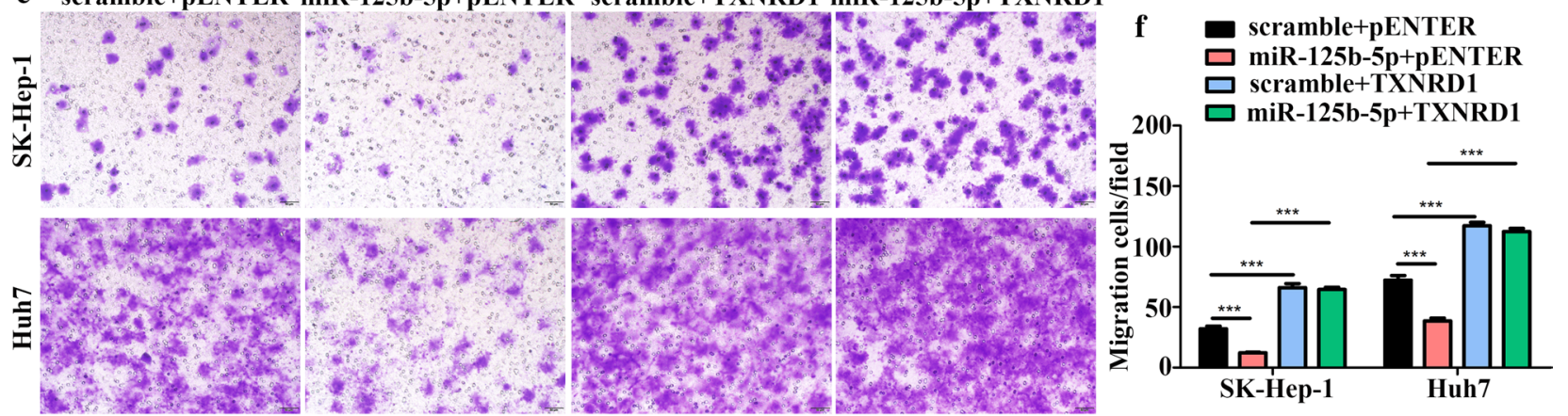

g scramble+pENTER miR-125b-5p+pENTER scramble+TXNRD1 miR-125b-5p+TXNRD1
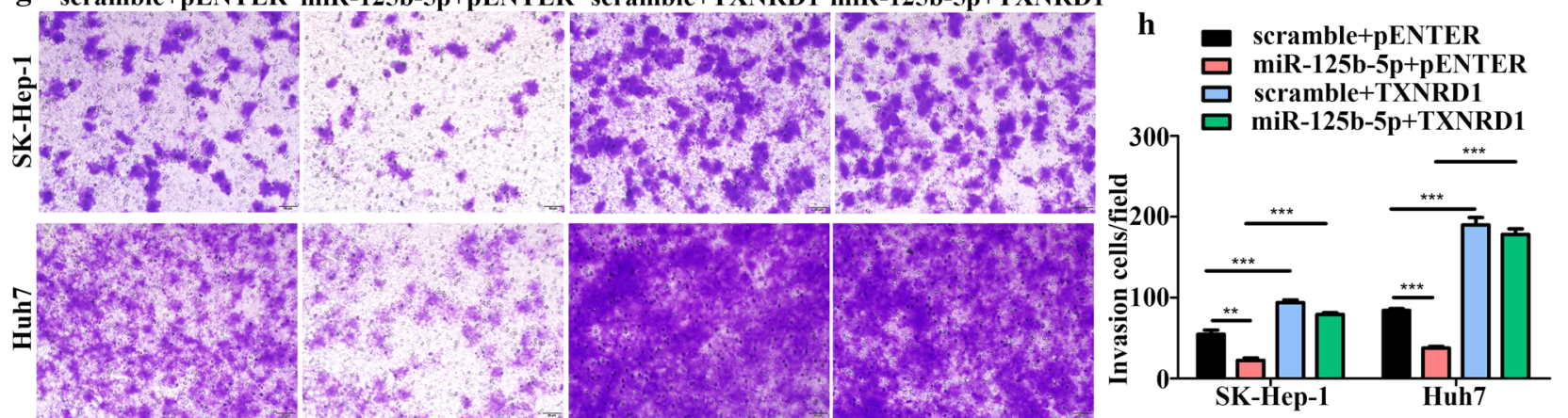

Fig. 5 TXNRD1 overexpression counteracts miR-125b-5p-mediated attenuation of HCC cell migration and invasion. a qRT-PCR- and $\mathbf{b}$ western blotting-based confirmation of miR-125b-5p and TXNRD1 overexpression. pENTER (empty plasmid) was used as a control to determine TXNRD1 overexpression ( $n=3$; one-way ANOVA and Dunnett's multiple comparison test). $\mathbf{c}-\mathbf{h}$ TXNRD1 overexpression abolished miR-125b-5p-mediated repression of $\mathbf{c}$, $\mathbf{d}$ HCC cell proliferation according to EdU-incorporation assay ( $n=3$; one-way ANOVA and Dunnett's multiple comparison test) and e-h migration and invasion in HCC cells $\left(n=3\right.$; one-way ANOVA and Dunnett's multiple comparison test). ${ }^{*} P<0.05$; ${ }^{* *} P<0.01 ;{ }^{* * *} P<0.001$ 


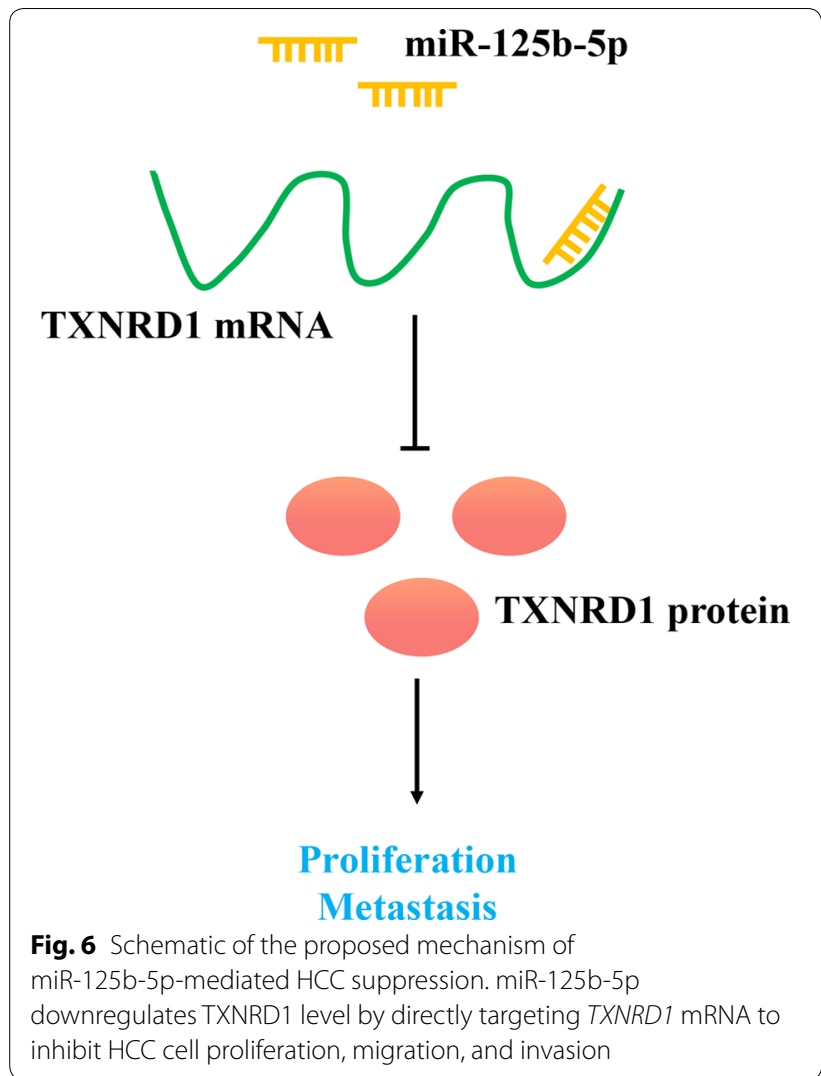

the attenuated expression of miR-125b-5p in HCC cells remains to be investigated in future research.

\section{Conclusions}

This represents the first study reporting that TXNRD1 is directly targeted by miR-125b-5p. Our results indicated that miR-125b-5p represents a tumor suppressor in HCC through its attenuation of TXNRD1 levels, thereby suggesting this miRNA as a potential target for HCC treatment.

\section{Additional file}

Additional file 1: Table S1. Primer sequences used in this study.

\section{Abbreviations}

CCK-8: cell counting kit-8; EDU: 5-ethynyl-2'-deoxyuridine; FBS: fetal bovine serum; GEO: gene expression omnibus; GSEA: gene set enrichment analysis; HCC: hepatocellular carcinoma; miRNAs: microRNAs; qRT-PCR: quantitative reverse transcription polymerase chain reaction; TCGA: The Cancer Genome Atlas; TRX1: thioredoxin 1; TXNRD1: thioredoxin reductase 1; UTR: untranslated region.

\section{Acknowledgements}

Not applicable.

\section{Authors' contributions}

SH designed and performed the experiments, analyzed the data, and drafted the paper. $Y Q$ and $M Z$ revised the paper. All authors read and approved the final manuscript.

\section{Funding}

This work was supported by the National Key Research and Development Program of China (No. 2017YFA0205200), National Natural Science Foundation of China (No. 81801811, 81571785, 81771957, and 81502642), China postdoctoral Science Foundation (No. 2018M631039), and Natural Science Foundation of Guangdong Province, China (No. 2016A030311055, 2016A030313770, and 2018A030313074).

\section{Availability of data and materials}

The datasets used and/or analyzed in this study are available from the corresponding author upon reasonable request.

\section{Ethics approval and consent to participate}

This study was approved by the Institutional Review Board of Zhuhai People's Hospital.

\section{Consent for publication}

Not applicable.

\section{Competing interests}

The authors declare that they have no competing interests.

Received: 27 March 2019 Accepted: 22 July 2019

Published online: 30 July 2019

\section{References}

1. Bray F, Ferlay J, Soerjomataram I, Siegel RL, Torre LA, Jemal A. Global cancer statistics 2018: GLOBOCAN estimates of incidence and mortality worldwide for 36 cancers in 185 countries. CA Cancer J Clin. 2018;68:394-424

2. Dang H, Takai A, Forgues M, Pomyen Y, Mou H, Xue W, Ray D, Ha KCH, Morris QD, Hughes TR, Wang XW. Oncogenic activation of the RNA binding protein NELFE and MYC signaling in hepatocellular carcinoma. Cancer Cell. 2017;32(101-14):e108.

3. Han TS, Voon DC, Oshima H, Nakayama M, Echizen K, Sakai E, Yong ZWE, Murakami K, Yu L, Minamoto T, Ock CY, Jenkins BJ, Kim SJ, Yang HK, Oshima M. Interleukin 1 up-regulates microRNA 135b to promote inflammation-associated gastric carcinogenesis in mice. Gastroenterology. 2019;156(1140-55):e1144.

4. Bach DH, Hong JY, Park HJ, Lee SK. The role of exosomes and miRNAs in drug-resistance of cancer cells. Int J Cancer. 2017;141:220-30.

5. Wu MZ, Cheng WC, Chen SF, Nieh S, O'Connor C, Liu CL, Tsai WW, Wu CJ, Martin L, Lin YS, Wu KJ, Lu LF, Izpisua Belmonte JC. miR-25/93 mediates hypoxia-induced immunosuppression by repressing cGAS. Nat Cell Biol. 2017;19:1286-96.

6. Rupaimoole R, Slack FJ. MicroRNA therapeutics: towards a new era for the management of cancer and other diseases. Nat Rev Drug Discov. 2017;16:203-22.

7. Yan W, Wu X, Zhou W, Fong MY, Cao M, Liu J, Liu X, Chen CH, Fadare O, Pizzo DP, Wu J, Liu L, Liu X, Chin AR, Ren X, Chen Y, Locasale JW, Wang SE. Cancer-cell-secreted exosomal miR-105 promotes tumour growth through the MYC-dependent metabolic reprogramming of stromal cells. Nat Cell Biology. 2018;20:597-609.

8. Arner ES. Focus on mammalian thioredoxin reductases_-important selenoproteins with versatile functions. Biochim Biophys Acta. 2009;1790:495-526

9. Peng X, Gimenez-Cassina A, Petrus P, Conrad M, Ryden M, Arner ES. Thioredoxin reductase 1 suppresses adipocyte differentiation and insulin responsiveness. Sci Rep. 2016;6:28080.

10. Lee D, Xu IM, Chiu DK, Leibold J, Tse AP, Bao MH, Yuen VW, Chan CY, Lai RK, Chin DW, Chan DF, Cheung TT, Chok SH, Wong CM, Lowe SW, Ng IO, Wong CC. Induction of oxidative stress through inhibition of thioredoxin reductase 1 is an effective therapeutic approach for hepatocellular carcinoma. Hepatology. 2019;69:1768-86. 
11. Stafford WC, Peng X, Olofsson MH, Zhang X, Luci DK, Lu L, Cheng Q, Trésaugues L, Dexheimer TS, Coussens NP, Augsten M, Ahlzén HM, Orwar O, Östman A, Stone-Elander S, Maloney DJ, Jadhav A, Simeonov A, Linder S, Arnér ESJ. Irreversible inhibition of cytosolic thioredoxin reductase 1 as a mechanistic basis for anticancer therapy. Sci Transl Med. 2018. https:// doi.org/10.1126/scitranslmed.aaf7444.

12. De Toni EN, Schlesinger-Raab A, Fuchs M, Schepp W, Ehmer U, Geisler F, Ricke J, Paprottka P, Friess H, Werner J, Gerbes AL, Mayerle J, Engel J. Age independent survival benefit for patients with hepatocellular carcinoma (HCC) without metastases at diagnosis: a population-based study. Gut. 2019. https://doi.org/10.1136/gutjnl-2018-318193.

13. Zhang L, Ge Y, Fuchs E. miR-125b can enhance skin tumor initiation and promote malignant progression by repressing differentiation and prolonging cell survival. Genes Dev. 2014;28:2532-46.

14. Yang D, Zhan M, Chen T, Chen W, Zhang Y, Xu S, Yan J, Huang Q, Wang J. miR-125b-5p enhances chemotherapy sensitivity to cisplatin by downregulating $\mathrm{Bcl} 2$ in gallbladder cancer. Sci Rep. 2017;7:43109.

15. Venkatadri R, Muni T, lyer AK, Yakisich JS, Azad N. Role of apoptosis-related miRNAs in resveratrol-induced breast cancer cell death. Cell Death Dis. 2016;7:e2104

16. Morelli E, Leone E, Cantafio ME, Di Martino MT, Amodio N, Biamonte L, Gullà A, Foresta U, Pitari MR, Botta C, Rossi M, Neri A, Munshi NC, Anderson KC, Tagliaferri P, Tassone P. Selective targeting of IRF4 by synthetic microRNA-125b-5p mimics induces anti-multiple myeloma activity in vitro and in vivo. Leukemia. 2015;29:2173-83.

17. Zhou JN, Zeng Q, Wang HY, Zhang B, Li ST, Nan X, Cao N, Fu CJ, Yan XL, Jia YL, Wang JX, Zhao AH, Li ZW, Li YH, Xie XY, Zhang XM, Dong Y, Xu YC, He LJ, Yue W, Pei XT. MicroRNA-125b attenuates epithelial-mesenchymal transitions and targets stem-like liver cancer cells through small mothers against decapentaplegic 2 and 4. Hepatology. 2015;62:801-15.

18. Kim JK, Noh JH, Jung KH, Eun JW, Bae HJ, Kim MG, Chang YG, Shen Q, Park WS, Lee JY, Borlak J, Nam SW. Sirtuin7 oncogenic potential in human hepatocellular carcinoma and its regulation by the tumor suppressors miR-125a-5p and miR-125b. Hepatology. 2013;57:1055-67.

19. Alpini G, Glaser SS, Zhang JP, Francis H, Han Y, Gong J, Stokes A, Francis T, Hughart N, Hubble L, Zhuang SM, Meng F. Regulation of placenta growth factor by microRNA-125b in hepatocellular cancer. J Hepatol. 2011;55:1339-45.

20. Fan DN, Tsang FH, Tam AH, Au SL, Wong CC, Wei L, Lee JM, He X, Ng IO, Wong $\mathrm{CM}$. Histone lysine methyltransferase, suppressor of variegation 3-9 homolog 1, promotes hepatocellular carcinoma progression and is negatively regulated by microRNA-125b. Hepatology. 2013;57:637-47.
21. Liang L, Wong CM, Ying Q, Fan DN, Huang S, Ding J, Yao J, Yan M, Li J, Yao M, Ng IO, He X. MicroRNA-125b suppressed human liver cancer cell proliferation and metastasis by directly targeting oncogene LIN28B2. Hepatology. 2010;52:1731-40.

22. Song S, Yang Y, Liu M, Liu B, Yang X, Yu M, Qi H, Ren M, Wang Z, Zou J, Li F, Du X, Zhang H, Luo J. MiR-125b attenuates human hepatocellular carcinoma malignancy through targeting SIRT6. Am J Cancer Res. 2018;8:993-1007

23. Degli Esposti D, Aushev VN. miR-500a-5p regulates oxidative stress response genes in breast cancer and predicts cancer survival. Sci Rep. 2017;7:15966.

24. Raninga PV, Di Trapani G, Vuckovic S, Tonissen KF. TrxR1 inhibition overcomes both hypoxia-induced and acquired bortezomib resistance in multiple myeloma through NF-small ka, Cyrillicbeta inhibition. Cell Cycle. 2016;15:559-72.

25. Xiaobo C, Majidi M, Feng M, Shao R, Wang J, Zhao Y, Baladandayuthapani V, Song J, Fang B, Ji L, Mehran R, Roth JA. TUSC2 (FUS1)-erlotinib induced vulnerabilities in epidermal growth factor receptor (EGFR) wild-type non-small cell lung cancer (NSCLC) targeted by the repurposed drug auranofin. Sci Rep. 2016;6:35741.

26. Zou P, Xia Y, Ji J, Chen W, Zhang J, Chen X, Rajamanickam V, Chen G, Wang Z, Chen L, Wang Y, Yang S, Liang G. Piperlongumine as a direct TrxR1 inhibitor with suppressive activity against gastric cancer. Cancer Lett. 2016:375:114-26.

27. Cho SY, Kim S, Son MJ, Rou WS, Kim SH, Eun HS. Clinical significance of the thioredoxin system and thioredoxin-domain-containing protein family in hepatocellular carcinoma. Dig Dis Sci. 2018;64:123-36.

28. Fu B, Meng W, Zeng X, Zhao H, Liu W. TXNRD1 is an unfavorable prognostic factor for patients with hepatocellular carcinoma. Biomed Res Int 2017;2017:4698167.

29. Boschi-Muller S, Branlant G. Methionine sulfoxide reductase: chemistry, substrate binding, recycling process and oxidase activity. Bioorg Chem. 2014;57:222-30.

30. Muri J, Heer S, Matsushita M, Pohlmeier L, Tortola L, Fuhrer T. The thioredoxin-1 system is essential for fueling DNA synthesis during T-cell metabolic reprogramming and proliferation. Nat Commun. 2018;9:1851.

\section{Publisher's Note}

Springer Nature remains neutral with regard to jurisdictional claims in published maps and institutional affiliations.
Ready to submit your research? Choose BMC and benefit from:

- fast, convenient online submission

- thorough peer review by experienced researchers in your field

- rapid publication on acceptance

- support for research data, including large and complex data types

- gold Open Access which fosters wider collaboration and increased citations

- maximum visibility for your research: over $100 \mathrm{M}$ website views per year

At BMC, research is always in progress.

Learn more biomedcentral.com/submissions 